\title{
Correction to: Re-fracture and correlated risk factors in patients with osteoporotic vertebral fractures
}

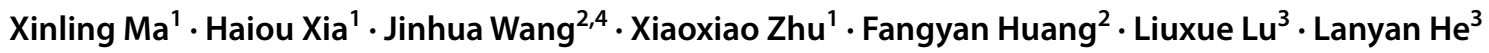

Published online: 2 April 2019

(c) Springer Japan KK, part of Springer Nature 2019

\section{Correction to: Journal of Bone and Mineral Metabolism https://doi.org/10.1007/s00774-018-0974-4}

In the Original publication of the article, the funding ID has been incorrectly published as (815602390) in the Acknowledgements. The correct Funding ID is provided below:

Acknowledgements This research was supported by the Fuxing Nursing Scientific Research Fund of Fudan University (FNF201708) and the National Natural Science Foundation of China (81560239). The research group thanks the patients who participated in this study. In addition, we thank Zhang Xianyan, Huang Chengjing, He Shiyuan, and Lv Shouqiang of Youjiang Medical University for Nationalities, who assisted with the study.

The original article can be found online at https://doi.org/10.1007/ s00774-018-0974-4.

Haiou Xia

hsxia@fudan.edu.cn

1 School of Nursing, Fudan University, Shanghai 200032, China

2 Youjiang Medical University for Nationalities, Baise 533000, China

3 The Affiliated Hospital of Youjiang Medical University for Nationalities, Baise 533000, China

4 West China School of Basic Medical Sciences and Forensic Medicine, Sichuan University, Sichuan 610041, China
Publisher's Note Springer Nature remains neutral with regard to jurisdictional claims in published maps and institutional affiliations. 